\title{
Molecular Twisting Power chiraler Azobenzole *
}

\author{
G. Heppke, F. Oestreicher und G. Scherowsky \\ Fachbereich Synthetische und Analytische Chemie der Technischen Universität Berlin
}

Z. Naturforsch. 37a, 1176-1184 (1982); eingegangen am 10. Juli 1982

Herrn Prof. Dr. E. Lippert zum 60. Geburtstag gewidmet

Molecular Twisting Power of Chiral Azobenzenes

The Molecular Twisting Power (MTP) of the trans- and cis-azocompounds 1-4, each substituted either with para- or orthostanding chiral side chains, of the azoxyanalogues 5,6 and of the chiral nitrobenzenes 7, 8 dissolved in the nematic liquid crystal NP 1132 are investigated. For all various series, althoug there is a distinct change in the geometry of the aromatic part and the substitution pattern, the helical screw sense of the induced cholesteric phase alternates with the number of bonds between the chiral center and the aromatic ring system. For the orthosubstituted azobenzenes 3, 4 a reversible inversion of the helix during the cis-trans-isomerisation is observed. Most of the compounds with the chiral center in the $\alpha$-position to the aromatic ring system show anomalous behaviour of the MTP.

\section{Einleitung}

Chirale Verbindungen erzeugen in nematischen Flüssigkristallen cholesterische Strukturen. Enantiomere induzieren entgegengesetzten Helixdrehsinn, vergleichbar mit den entgegengesetzten optischen Drehwerten chiraler Antipoden in isotropen Lösungen. Bei kleinen Konzentrationen chiraler Verbindungen in Flüssigkristallen steigt der Reziprokwert der Helixganghöhe proportional zur Teilchendichte der chiralen Moleküle [1-3]. Der Proportionalitätsfaktor ist eine für die zugesetzten chiralen Moleküle charakteristische Größe. Auf diese Weise läßt sich die Molecular Twisting Power (MTP) definieren [2], deren Wert in gewissen Grenzen noch von der Wahl des nematischen Lösungsmittels abhängt.

Ähnlich wie für die optische Aktivität gibt es auch für die MTP keinen allgemeingültigen Zusammenhang mit der Molekülkonfiguration. Während für den Circulardichroismus und die optische Rotationsdispersion im wesentlichen das Elektronensystem des Moleküls verantwortlich ist [4], handelt es sich bei der Induktion cholesterischer Phasen um eine mechanische Verdrillung gegen elastische Rückstellkräfte des Flüssigkristalles [1], die demzufolge auch Aussagen über die Geometrie des induzierenden Moleküls machen sollte. Die Untersuchungsmethoden

* Teilweise vorgetragen auf der 7. Internationalen Flüssigkristallkonferenz in Bordeaux, Frankreich, 1978.

Reprint requests to Prof. G. Heppke, Fachbereich Synthetische und Analytische Chemie der Technischen Universität Berlin, Straße des 17. Juni 135, 1000 Berlin 12. stellen daher zwei unabhängige Wege der Chiralitätsbeobachtung dar.

Im Gegensatz zu den ausgedehnten Kenntnissen über die optische Aktivität ist weitaus weniger über die MTP chiraler Moleküle bekannt. Aus den bisherigen Untersuchungen konnten im wesentlichen zwei Regeln über den induzierten Helixdrehsinn abgeleitet werden, die sich auf chirale Verbindungen mit einem Chiralitätszentrum beziehen.

Die von Korte et al. angegebene Regel geht von der Raumerfüllung der Liganden aus [5]. Damit wird eine Rangordnung am Chiralitätszentrum und letztlich der Helixdrehsinn festgelegt. Eine große Anzahl Moleküle mit einem chiralen Zentrum lassen sich in dieses Schema einordnen, wobei allerdings Moleküle mit dem Chiralitätszentrum in Ringen sowie solche mit verzweigten Liganden oder Liganden, die den Wirtsmolekülen ähnlich sind, nicht durch eine Rangfolge klassifiziert werden können [5].

Die zweite Regel bezieht sich auf mesogene Systeme mit chiraler Seitenkette. So wurde von Gray und McDonnell [6] an Cyanobiphenylen mit chiraler Alkylkette

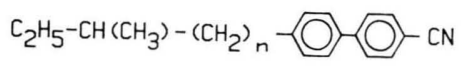

gefunden, daß der Drehsinn mit der Anzahl der $\mathrm{CH}_{2}$-Gruppen zwischen dem chiralen Zentrum und dem aromatischen Ringsystem alterniert. Dieser odd-even-Effect tritt auch auf, wie unabhängig davon an anderen mesogenen Verbindungen mit chiraler

0340-4811 / 82 / 1000-1176 $\$ 01.30 / 0$. - Please order a reprint rather than making your own copy. 
Seitenkette beobachtet wurde, wenn in der Kette zwischen dem chiralen Zentrum und dem Aromaten ein Sauerstoffatom [6, 7] oder eine Estergruppe [7] steht. Bezieht man allgemein auf die Zahl der Bindungen zwischen dem Aromaten und dem chiralen Zentrum, so sagt die odd-even-Vorzeichenregel $[6,7]$ beispielsweise bei S-Konfiguration und gerader Zahl der Bindungen eine rechtshändige Helix voraus.

Der odd-even-Effekt läßt sich nicht durch eine auf der Raumerfüllung beruhenden Rangfolge der Liganden beschreiben, denn bei der schrittweisen Verschiebung des chiralen Zentrums innerhalb der Alkylkette ändert sich diese Rangfolge nicht. Für den allgemeinen Fall einer chiralen Verbindung führen beide Regeln zwangsläufig zu widersprüchlichen Aussagen, woraus folgt, daß für die Gültigkeit der odd-even-Vorzeichenregel noch weitere zusätzliche Strukturelemente verantwortlich sein müssen.

Eine theoretische Erklärung des odd-even-Effektes wurde von van der Meer und Vertogen gegeben [8]. Darin wird vorausgesetzt, daß durch die Orientierung der molekularen Längsachse der chiralen Verbindung im Flüssigkristall eine Bezugsachse gegeben ist, bezüglich der man die Lage des chiralen Zentrums mit seinen unterschiedlichen Liganden und gerichteten Wechselwirkungen definieren kann. Das Einfügen einer weiteren $\mathrm{CH}_{2}$-Gruppe läßt das chirale Zentrum einen Schritt weiter in der gewinkelten Alkylkette wandern. Das entspricht einer Symmetrieoperation, die eine Umkehr des Vorzeichens der chiralen Wechselwirkung mit der Umgebung und damit eine Umkehr des Vorzeichens der induzierten Helix zur Folge hat.

Die bisherigen Untersuchungen des odd-evenEffektes beziehen sich auf mesogene Moleküle, deren Längsachse vor allem durch den aromatischen Mittelteil festgelegt wird, wohingegen die Raumerfüllungsregel ihre Gültigkeit eher für solche Moleküle gezeigt hat, bei denen keine bevorzugte Ordnung im Flüssigkristall zu erwarten ist. Ziel der vorliegenden Arbeit war es, zu prüfen, inwieweit Strukturänderungen am aromatischen Mittelteil, die zu einem Verlust der Mesogenität führen, die Gültigkeit der odd-even-Vorzeichenregel aufheben.

Dazu wurde das mit optisch aktiven Alkylketten substituierte Azobenzolsystem ausgewählt, das den Vergleich des Einflusses der Molekülstruktur sowohl zwischen para- und ortho-Substitution, als auch zwischen trans- und cis-Isomeren ermöglicht. Der Abstand des chiralen Zentrums zum aromatischen Ringsystem wurde dabei gleichermaßen systematisch variiert:

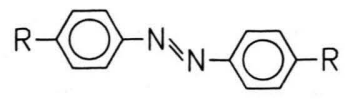

para-substituierte trans-Azobenzole (1a-d)

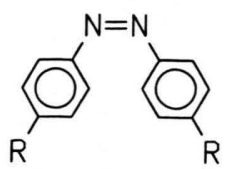

para-substituierte cis-Azobenzole (2a-d)

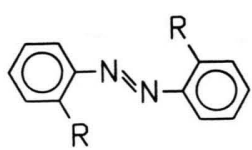

ortho-substituierte trans-Azobenzole (3a-d)

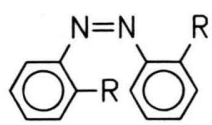

ortho-substituierte cis-Azobenzole (4a-d)

Darüber hinaus wurden die entsprechenden transAzoxyverbindungen in die Untersuchung miteinbezogen, um den Einfluß der stark polaren Azoxygruppe auf Größe und Vorzeichen der MTP zu ermitteln :

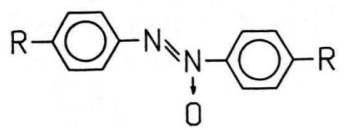

para-substituierte trans-Azoxybenzole (5a-d)

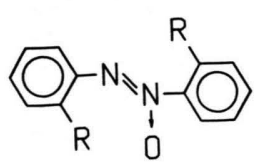

ortho-substituierte trans-Azoxybenzole $(6 \mathbf{a}-\mathbf{d})$

Weiterhin wurden die als Vorstufen bei der Synthese auftretenden Nitrobenzole mit para- bzw. ortho-ständigen Seitenketten untersucht:

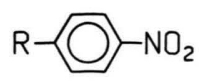

para-substituierte Nitrobenzole (7a-d) 
<smiles>[R]c1ccccc1[N+](=O)[O-]</smiles>

ortho-substituierte Nitrobenzole $(\mathbf{8} \mathbf{a}-\mathbf{d})$

In allen Verbindungstypen $\mathbf{1}-\mathbf{8}$ bedeutet $\mathrm{R}$

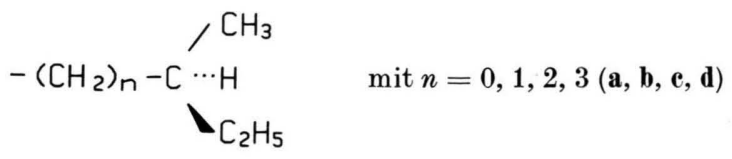

d. h. das chirale Zentrum, das einheitlich die S-Konfiguration aufweist, ist jeweils durch 0 bis $3 \mathrm{CH}_{2}$ Gruppen vom aromatischen Kern getrennt.

\section{Experimentelles}

Für die Untersuchungen standen insgesamt 32 Verbindungen der folgenden acht Gruppen zur Verfügung :

1. trans-4, $4^{\prime}$-Dialkylazobenzole $(1 \mathrm{a}-\mathrm{d})$

2. cis-4, $4^{\prime}$-Dialkylazobenzole $(\mathbf{2} \mathbf{a}-\mathbf{d})$

3. trans-2, $2^{\prime}$-Dialkylazobenzole $(3 \mathbf{a}-\mathbf{d})$

4. cis-2, $2^{\prime}$-Dialkylazobenzole $(4 \mathbf{a}-\mathbf{d})$

5. trans-4, $4^{\prime}$-Dialkylazoxybenzole $(\mathbf{5 a}-\mathbf{d})$

6. trans-2, 2'-Dialkylazoxybenzole $(\mathbf{6 a}-\mathbf{d})$

7. 4-Alkylnitrobenzole $(\mathbf{7} \mathbf{a}-\mathbf{d})$

8. 2-Alkylnitrobenzole $(\mathbf{8 a}-\mathbf{d})$

Die Synthese der Verbindungen ist an anderer Stelle ausführlich beschrieben [9].

Die Ganghöhe $p$ und die Händigkeit der durch die Verbindungen induzierten cholesterischen Phasen wurden an 1- bis 3-proz. Lösungen in der nematischen Phase NP 1132 (Merck) nach der modifizierten Grandjean-Cano-Methode bestimmt [7, 10]. Das aus der Ganghöhe $p$ und der Konzentration $c$ in Gew. $-\%$ des chiralen Dotierstoffes berechnete Produkt $p c$ erweist sich im untersuchten Konzentrationsbereich als Konstante. Um einen Vergleich der verschiedenen Dotierstoffe untereinander zu ermöglichen, wird auf gleiche Teilchenzahl pro Volumen bezogen. Die auf die Molarität $c_{\mathrm{M}}$ des chiralen Dotierstoffes bezogene Molecular Twisting Power (MTP) $\beta=1 / p c_{\mathrm{M}}$ mit der Einheit $\mathrm{m}^{2} / \mathrm{mol}$ ergibt sich dann aus $\beta=100 \mathrm{M} / p c \varrho$ worin $M$ die Molmasse des chiralen Dotierstoffes und $\varrho$ die Dichte der Lösung bedeuten, die näherungsweise gleich der Dichte des nematischen Flüssigkristalles NP 1132 mit $\varrho=$ $0,97 \mathrm{~g} / \mathrm{cm}^{3}$ gesetzt wird.

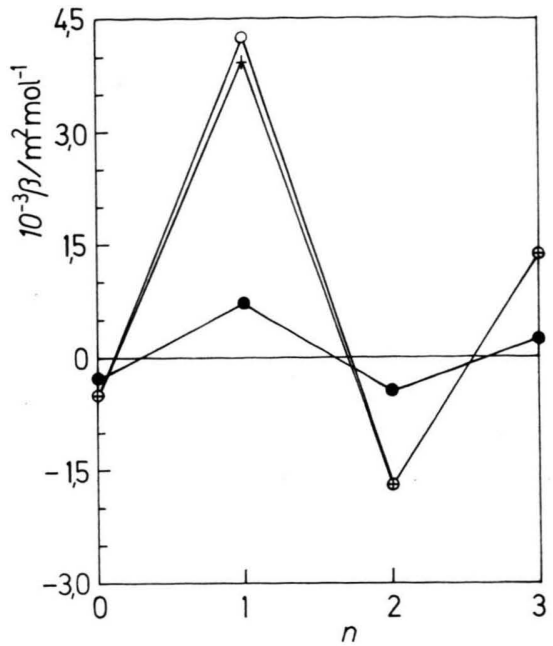

Abb. 1. Molecular Twisting Power $\beta$ der para-substituierten trans-Azobenzole 1a-d ( 1 ), der entsprechencen Azoxybenzole 5a-d (+) und der para-substituierten Nitrobenzole 7a-d (•) in Abhängigkeit von der Zahl $n$ der $\mathrm{CH}_{2}$-Gruppen zwischen dem chiralen Zentrum und dem aromatischen Ringsystem.

\section{Ergebnisse und Diskussion}

\subsection{Trans-konfigurierte Verbindungen}

In Abb. 1 sind die MTP-Werte, bestimmt in NP 1132, als Funktion der Anzahl $n$ der $\mathrm{CH}_{2}$-Gruppen zwischen dem chiralen Zentrum und dem aromatischen Ringsystem für die para-substituierten trans-Azo und Azoxyverbindungen $(\mathbf{1} \mathbf{a}-\mathbf{d}$ und

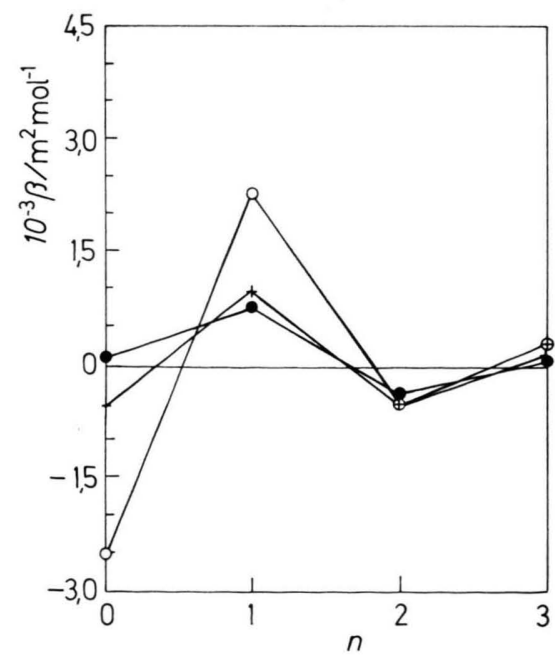

Abb. 2. Molecular Twisting Power $\beta$ der ortho-substituierten trans-Azobenzole $\mathbf{3 a - d}(0)$, der entsprechenden Azoxybenzole 6a-d $(+)$ und der ortho-substituierten Nitrobenzole $8 \mathbf{a}-\mathbf{d}(\bullet)$. 
5a-d) dargestellt. Zum Vergleich sind die entsprechenden para-substituierten Nitroverbindungen ( 7 a -d) eingetragen. Abbildung 2 enthält MTP. Werte der entsprechenden ortho-substituierten Verbindungen ( $3 \mathbf{a}-\mathbf{d}, 6 \mathbf{a}-\mathbf{d}$ und $8 \mathbf{a}-\mathbf{d})$.

\subsubsection{Helixdrehsinn}

Sowohl die para- als auch die ortho-substituierten Azo- und Azoxyverbindungen zeigen ein Alternieren des Vorzeichens der Helixganghöhe. Der Helixdrehsinn gehorcht der odd-even-Vorzeichenregel $[6,7]$, die beispielsweise für eine gerade Anzahl Bindungen zwischen aromatischem Ringsystem und dem chiralen Zentrum im Falle der S-Konfiguration des chiralen Kohlenstoffatoms eine Rechtsschraube $(p>0)$ vorhersagt.

Im Gegensatz dazu sollten nach der Raumerfüllungsregel [5] alle hier beschriebenen Verbindungen $1 \mathbf{a}-\mathbf{d}$ bis $8 \mathbf{a}-\mathbf{d}$ einheitlich eine linkshändige Helix induzieren. Während es nicht überraschend ist, $\mathrm{da} ß$ die linear gebauten para-substituierten Verbindungen der an mesogenen Systemen gefundenen oddeven-Vorzeichenregel gehorchen, war dies für die gewinkelt gebauten ortho-substituierten Verbindungen nicht von vornherein zu erwarten. Ebenso erstaunlich ist, daß auch die kürzeren Nitroverbindungen nicht nur ein Alternieren der MTP zeigen, sondern bis auf die Ausnahme 8 a sogar der odd-even-Vorzeichenregel genügen, obwohl diese Verbindungen nicht einmal potentiell mesogen sind. Die Sonderstellung der ortho-substituierten Alkylnitroverbindung $8 \mathbf{a}$ mit zum Aromaten $\alpha$-ständigem chiralen Zentrum ist vermutlich auf die starke sterische Behinderung zwischen der Nitrogruppe und der chiralen Alkylkette zurückzuführen. Die Rechtshändigkeit der durch 8 a induzierten Helix widerspricht interessanterweise auch der Voraussage nach der Raumerfüllungsregel.

\subsubsection{Größe der M T P - Werte}

\subsubsection{Para-substituierte Verbindungen}

Betrachtet man die Absolutwerte der MTP in Abb. 1 und Tab. 1, so ist zu erkennen, daß die para-substituierten Azo- und Azoxyverbindungen ( $1 \mathbf{a}-\mathbf{d}$ und $5 \mathbf{a}-\mathbf{d})$ bei gleicher chiraler Seitenkette nahezu identische MTP-Werte haben. Es ist zu vermuten, daß diese Übereinstimmung auf der Gleichheit des Ordnungsgrades der beiden Verbindungstypen beruht.

Die para-substituierten Verbindungen mit $n=1,2$ und $3(\mathbf{1} \mathbf{b}-\mathbf{d}$ und $\mathbf{5} \mathbf{b}-\mathbf{d})$ zeigen die auch schon an anderen mesogenen Verbindungen beobachtete stetige Abnahme der Beträge der MTP mit zunehmender Entfernung des chiralen Zentrums vom aromatischen Ringsystem. Dies wird nach dem Modell von van der Meer und Vertogen durch die Abnahme der chiralen Wechselwirkung mit der flüssigkristallinen Umgebung infolge der Zunahme der Flexibilität der Alkylkette an der Stelle des chiralen Zentrums erklärt [8]. Hiernach war für die Verbindungen mit dem chiralen Zentrum direkt am Aromaten $(n=0)$ ein besonders hoher Wert der MTP zu erwarten. Da bisher keine derartige Verbindung bekannt war, schien auch aus diesem Grunde der nötige synthetische Aufwand zur Darstellung dieser Verbindungen unter Einbeziehung einer Enantiomerentrennung gerechtfertigt [9]. Im Gegensatz zu den Erwartungen zeigen die para-substituierten Azound Azoxyverbindungen 1 a bzw. 5 a eine unerwartet kleine MTP; die ortho-substituierte trans-Azoverbindung 3 a hingegen weist tatsächlich den größten MTP-Wert innerhalb der Homologen auf (siehe Abbildung 4).

In Abb. 1 sind neben den MTP-Werten der Azound Azoxyverbindungen auch die der entsprechenden para-substituierten Nitroverbindungen $7 \mathbf{a}-\mathbf{d}$

Tab. 1. $p c$-Produkte und Molecular Twisting Power $\beta$ in NP 1132 bei $25^{\circ} \mathrm{C}$.

\begin{tabular}{|c|c|c|c|c|c|c|c|c|}
\hline & \multicolumn{2}{|l|}{$\mathbf{a}$} & \multicolumn{2}{|l|}{ b } & \multicolumn{2}{|l|}{ e } & \multicolumn{2}{|l|}{ d } \\
\hline & $\begin{array}{l}p c \\
\mu \mathrm{m} \text { Gew.- } \%\end{array}$ & $\begin{array}{l}\beta \\
\mathrm{m}^{2} \mathrm{~mol}^{-1}\end{array}$ & $\begin{array}{l}p c \\
\mu \mathrm{m} \text { Gew.- } \%\end{array}$ & $\begin{array}{l}\beta \\
\mathrm{m}^{2} \mathrm{~mol}^{-1}\end{array}$ & $\begin{array}{l}p c \\
\mu \mathrm{m} \text { Gew.- } \%\end{array}$ & $\begin{array}{l}\beta \\
\mathrm{m}^{2} \mathrm{~mol}^{-1}\end{array}$ & $\begin{array}{l}p c \\
\mu \mathrm{m} \text { Gew.- } \%\end{array}$ & $\begin{array}{l}\beta \\
\mathrm{m}^{2} \mathrm{~mol}^{-1}\end{array}$ \\
\hline 1 & $-64,8$ & -470 & $+7,8$ & +4240 & $-21,2$ & -1700 & $\begin{array}{r}28,8 \\
\end{array}$ & +1350 \\
\hline 3 & $-12,1$ & -2500 & $+14,6$ & +2280 & $-71,2$ & -510 & $\begin{array}{r}135,5 \\
\end{array}$ & $\begin{array}{r}290 \\
+\quad 290\end{array}$ \\
\hline 5 & $-63,6$ & -500 & $\begin{array}{l}+8,8 \\
\end{array}$ & +3950 & $-22,3$ & -1690 & $+30,0$ & +1360 \\
\hline 6 & $-57,6$ & -560 & $+34,9$ & +1000 & $-72,2$ & -520 & $+248,7$ & +160 \\
\hline 7 & $-64,3$ & -290 & $\begin{array}{r}+27,5 \\
\end{array}$ & $\begin{array}{r}+\quad 720 \\
+\end{array}$ & $-47,8$ & -450 & $+101,0$ & $\begin{array}{r}+230 \\
+\quad 2\end{array}$ \\
\hline 8 & $+182,0$ & +100 & $+25,6$ & +780 & $-56,1$ & -380 & $+310,1$ & + \\
\hline
\end{tabular}


eingetragen. Für einen sinnvollen Vergleich ist zu berücksichtigen, daß die Nitroverbindungen nur ein chirales Zentrum pro Molekül enthalten. Auch bei Bezug auf gleiche Dichte chiraler Zentren, d. h. Verdopplung der MTP-Werte der in Abb. 1 und Tab. 1 angegebenen MTP der Nitroverbindungen liegen die Werte noch erheblich unter denen der Azo- und Azoxyverbindungen. Dieser Unterschied dürfte auf den geringeren Ordnungsgrad des kürzeren Alkylnitrobenzolmoleküls gegenüber dem alkylsubstituierten Azobenzolsystem zurückzuführen sein. Die para-substituierte Nitroverbindung 7 a mit dem chiralen Zentrum direkt am Aromaten nimmt eine Sonderstellung ein, insofern, als ihr doppelter MTP. Wert über dem MTP-Wert der entsprechenden Azound Azoxyverbindung liegt.

\subsubsection{Ortho-substituierte Verbindungen}

Ein Vergleich der Molekülgeometrie der paraund ortho-substituierten Verbindungen anhand von Kalottenmodelleen zeigt, daß bei den ortho-substituierten Verbindungen im Gegensatz zu den parasubstituierten Verbindungen eine Moleküllängsachse nur schwer zu definieren ist und bei den Azo- und Azoxyverbindungen nicht notwendigerweise an die Hauptachse des mesogenen Mittelteiles gebunden ist. Insofern ist es erstaunlich, daß auch diese Verbindungen (mit Ausnahme der Nitroverbindung 8 a) ein Alternieren der MTP zeigen. Für die orthosubstituierte Azoverbindung 3 a mit $n=0$ findet man einen außergewöhnlich hohen Wert der MTP, der nicht nur über dem der entsprechenden parasubstituierten Verbindung 1 a liegt, sondern darüber hinaus den höchsten Wert in der Reihe der orthosubstituierten Verbindungen darstellt.

Vergleicht man die MTP-Werte aller hier untersuchten chiralen Verbindungen, die das Asymmetriezentrum in $\alpha$-Stellung zum aromatischen Kern $(n=0)$ haben, mit ihren Homologen, so erkennt man, daß sie hinsichtlich Größe und Vorzeichen der MTP stets eine Sonderstellung einnehmen. Diese Sonderstellung wird vermutlich durch eine Einschränkung der konformativen Beweglichkeit durch den Aromaten als Liganden am chiralen Zentrum bewirkt.

Der im Gegensatz zu den para-substituierten Verbindungen (Abb. 1) bei den ortho-substituierten Verbindungen (Abb. 2) auftretende Unterschied der MTP-Werte zwischen Azo- und Azoxyverbindungen hängt möglicherweise damit zusammen, daß bei den ortho-substituierten Verbindungen transoide und cisoide Konformere bezüglich der Lage der Alkylketten der beiden substituierten Aromaten zueinander vorliegen können. Die Azoxygruppe könnte hier zu einer energetischen Bevorzugung des cisoiden Konformeren führen, das dementsprechend die vergleichsweise niedrigere MTP aufweisen sollte.

\subsection{Cis-trans-Isomerie}

Einer der wesentlichen Gesichtspunkte dieser Arbeit lag in der Frage, inwieweit sich die cis- und trans-Isomeren der substituierten Azobenzole in ihrer MTP unterscheiden.

Um die reinen cis-Isomeren untersuchen zu können, wurde bei der Probenvorbereitung möglichst unter Lichtausschluß gearbeitet. Jedoch zeigte sich, daß sowohl die thermische Isomerisierung bei Raumtemperatur als auch die photochemische, bedingt durch die notwendige Mikroskopbeleuchtung, eine Verminderung des cis-Anteiles in der Probe während der Messung bewirkte. Da sich der Reziprokwert der gemessenen Ganghöhe additiv aus den reziproken Ganghöhen der cis- und trans-Anteile zusammensetzt, verursacht der entstehende trans-Anteil eine Verschiebung der Meßwerte in Richtung der $\beta$-Werte der trans-Verbindungen. Die in den Abb. 3 und 4 eingetragenen Meßpunkte der cis-Verbindungen stellen daher nur Näherungswerte dar. Die Pfeile kennzeichnen dementsprechend die Richtung, in der die $\beta$-Werte der reinen cis-Verbindungen liegen sollten.

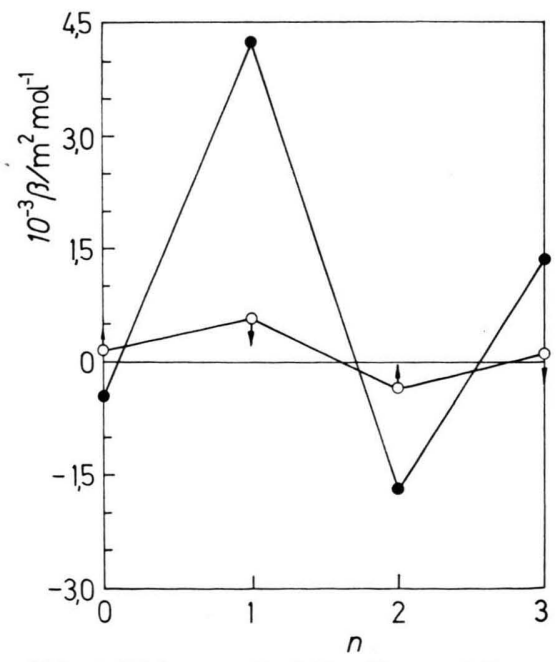

Abb. 3. Molecular Twisting Power $\beta$ der para-substituierten trans-Azobenzole 1a-d (•) und der para-substituierten cis-Azobenzole $\mathbf{2} \mathbf{a}-\mathbf{d}(0)$. 


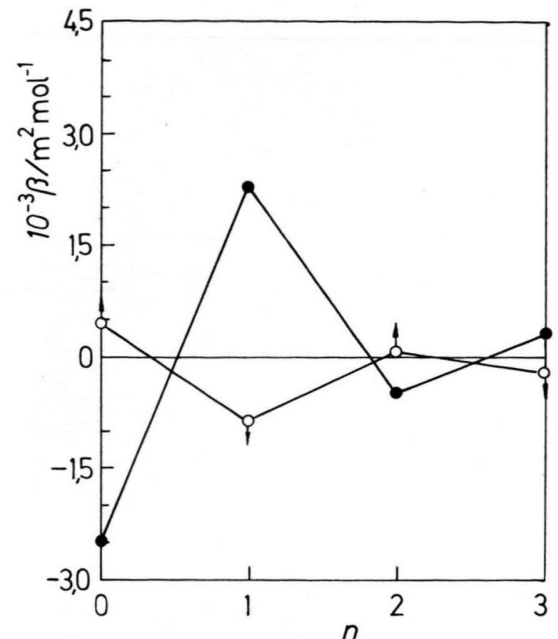

Abb. 4. Molecular Twisting Power $\beta$ der ortho-substituierten trans-Azobenzole $\mathbf{3 a - d}(\bullet)$ und der ortho-substituierten cis-Azobenzole 4a-d (0).

\subsubsection{Para-substituierte cis- \\ Verbindungen}

Bei den para-substituierten cis-Azobenzolen (Abb. 3) zeigen die Homologen mit $n=1,2$ und 3 (2 b-d) gegenüber den entsprechenden trans-Verbindungen erheblich kleinere MTP-Werte, die aber jeweils das gleiche Vorzeichen besitzen, d.h. diese cis-Verbindungen gehorchen ebenfalls der odd-evenVorzeichenregel.

Dagegen induziert die Verbindung 2 a $(n=0)$ entgegen der Voraussage eine Rechtsschraube während die trans-Verbindung im Einklang mit der Vorzeichenregel eine Linksschraube induziert. Das bedeutet, daß trotz gleichbleibender Konfiguration der beiden chiralen Zentren allein durch Änderung der Konfiguration an der Azogruppe (cis-trans-Isomerisierung) eine Umkehr des Drehsinnes der im Flüssigkristall induzierten Helix auftritt.

\subsubsection{Ortho-substituiertecis - Verbindungen}

Bei den ortho-substituierten Azoverbindungen (Abb. 4) zeigen alle untersuchten Homologen dieses Phänomen einer Inversion des Helixdrehsinnes bei der cis-trans-Isomerisierung. Der Helixdrehsinn aller ortho-substituierten cis-Verbindungen weist also, wie Abb. 4 zeigt, ebenfalls ein odd-even-Verhalten auf, allerdings mit entgegengesetztem Vorzeichen.

\subsubsection{Helixinversion}

Die cis- und trans-Isomeren der Azobenzole lassen sich auch auf photochemischem Wege ineinander überführen [11], was eine direkte Beobachtung der Umkehr des Helixdrehsinnes bei der Isomerisierung ermöglicht. Ausgehend von den Absorptionsspektren der substituierten Azobenzole [9], die dem Spektrum des unsubstituierten Azobenzols sehr ähnlich sind, wurde die durch ein Interferenzfilter selektierte Spektrallinie der Wellenlänge $\lambda_{\max }=334 \mathrm{~nm}$ einer Quecksilberhochdrucklampe (HBO $100 \mathrm{~W} / 2$ ) ausgewählt. Für diese Wellenlänge läßt sich im photochemischen Gleichgewicht eine 80- bis 90-proz. Anreicherung der cis-Verbindung abschätzen.

Mit dieser Lichtquelle wurden 1 -3-proz. Lösungen der ortho-substituierten Azobenzole in NP 1132 im auf $0{ }^{\circ} \mathrm{C}$ thermostatisierten Mikroskopheiztisch bestrahlt. Dabei wurde die in einer früheren Arbeit beschriebene Probenanordnung verwendet [10], bei der die Grandjean-Cano-Disklinationslinien infolge der besonderen Oberflächenpräparation Spiralen ausbilden, aus deren Drehsinn sich der Helixdrehsinn der cholesterischen Phase ablesen läßt.

Nach mehrstündiger Bestrahlung konnte bei allen ortho-substituierten Azobenzolen eine Umkehr des Drehsinnes der Disklinationsspiralen erreicht und damit die Umkehr des Helixdrehsinnes beim Übergang von den cis- zu den trans-konfigurierten chiralen Verbindungen ( $4 \mathrm{a}-\mathrm{d}$ bzw. $3 \mathrm{a}-\mathrm{d})$ nachgewiesen werden. Nach Abschalten der Lichtquelle und Erwärmen der Probe auf Raumtemperatur vollzieht sich die Rückreaktion in die trans-Verbindung unter erneuter Helixinversion.

Die Kinetik der Rückreaktion ist am Beispiel der ortho-substituierten Azoverbindung mit $n=1$ $(4 \mathbf{b} \rightarrow 3 \mathrm{~b})$ in den Abbildungen $5 \mathrm{a}-\mathrm{e}$ dargestellt. Die Konzentration in NP 1132 beträgt dabei 2,04 Gew.- $\%$. Abbildung 5 a gibt den $\mathrm{Zu}$ stand unmittelbar nach Abschalten der Strahlungsquelle wieder. Die Auswertung der (im mathematischen Sinne) linkshändigen Spirale ergibt eine Ganghöhe von $p=-58,4 \mu \mathrm{m}$. Die Rückbildung der trans-Verbindung bei Raumtemperatur wird vor allem durch die für die photographischen Aufnahmen notwendige starke Mikroskopbeleuchtung bewirkt. Daher wurde die Beleuchtung für die gesamte Zeit der Beobachtung konstant gehalten. Etwa 13 min später erfolgt die Vorzeichenumkehr der Helixschraube, d.h. in der Probe liegt eine nematische 


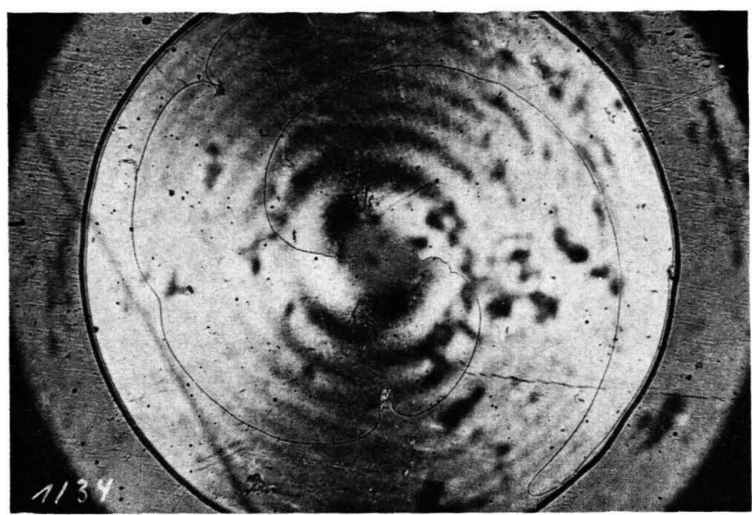

a)

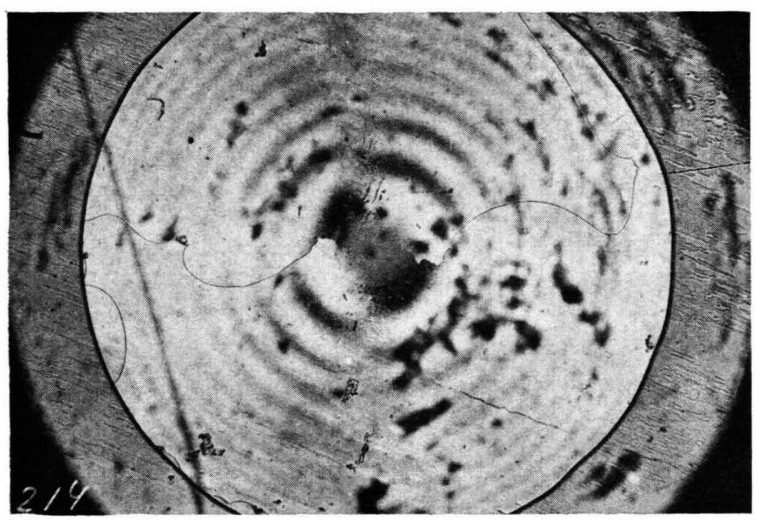

b)

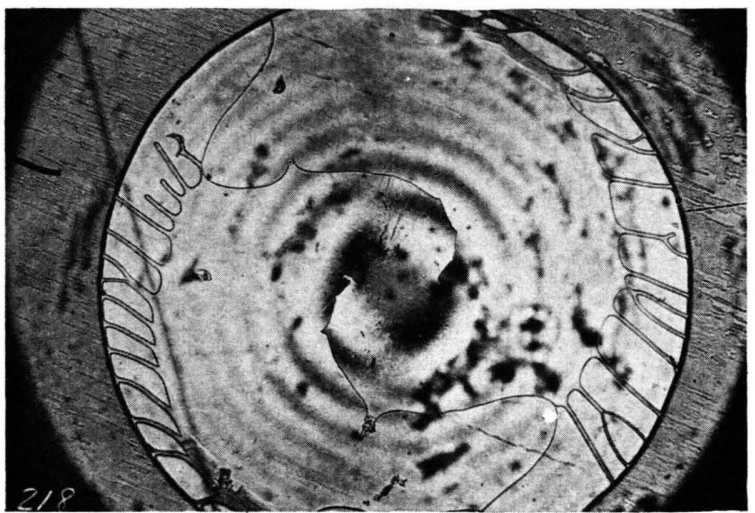

c)

Phase vor (Abbildung $5 \mathrm{~b}$ ). Nach $30 \mathrm{~min}$ hat sich bereits wieder eine rechtshändige Helixschraube mit der Ganghöhe $p=51,8 \mu \mathrm{m}$ ausgebildet, wie Abb. $5 \mathrm{c}$ zeigt. Die beiden weiteren Abbildungen ( $5 \mathrm{~d}$ und 5 e) geben einen Eindruck über den weiteren Fortgang der Kinetik, bis etwa nach 1000 min eine

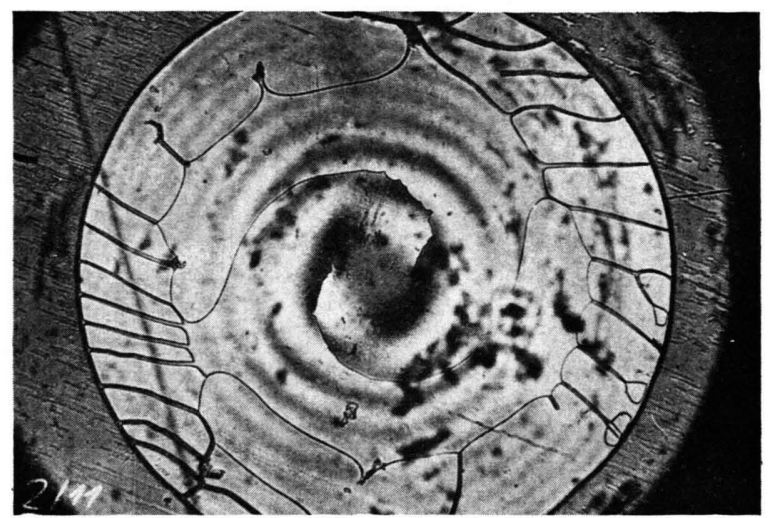

d)

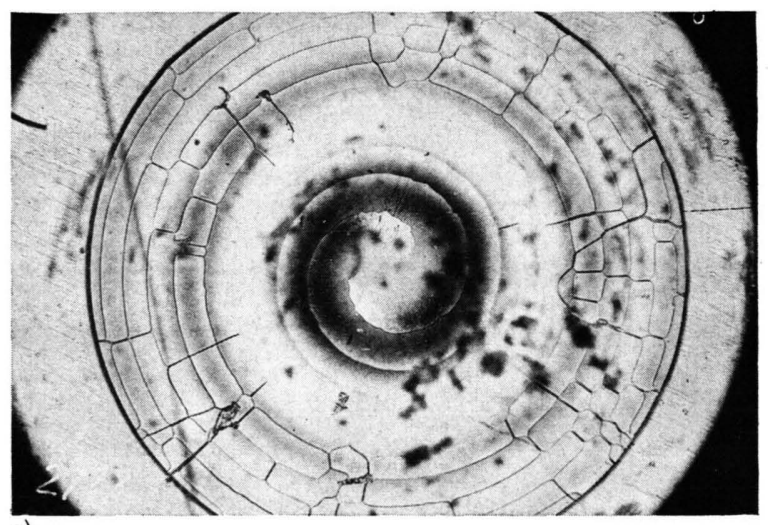

e)

Abb. 5a-e. Zeitliche Änderung der Disklinationsspiralen während der cis-trans-Isomerisierung der ortho-substituierten Azoverbindung 4b nach 3b
Abb. 5 a $t=0 \mathrm{~min}$,
Abb. $5 \mathrm{~b} \quad t=13 \mathrm{~min}$, $p=-58,4 \mu \mathrm{m}$

Abb. 5c $t=30 \mathrm{~min}$, $p=+51,8 \mu \mathrm{m}$.

Abb. $5 \mathrm{~d} \quad t=39 \mathrm{~min}$

Abb. 5e $t=980$ min,

$p=+29,9 \mu \mathrm{m}$. $p=+7,2 \mu \mathrm{m}$.

Ganghöhe von $7,2 \mu \mathrm{m}$ beobachtet wird, was nahezu der an der reinen trans-konfigurierten Verbindung $3 \mathbf{b}$ gemessenen MTP (Tab. 1) entspricht.

Die so bestimmten Ganghöhen sind in Abb. 6 in Abhängigkeit von der Zeit dargestellt. Für eine theoretische Beschreibung des Zeitgesetzes $p=p(t)$ 
wird angenommen, daß die Isomerisierung nach einer Reaktion erster Ordnung mit der Reaktionsgeschwindigkeitskonstante $k$ abläuft. Damit ergibt sich für die Ganghöhe $p$

$$
1 / p=1 / p_{\text {trans }}+\left(c_{\text {cis }}^{o} / c\right)\left(1 / p_{\text {cis }}-1 / p_{\text {trans }}\right) \exp (-k t) \text {. }
$$

Darin bedeuten $p_{\text {trans }}$ bzw. $p_{\text {cis }}$ jeweils die Grenzwerte der Ganghöhe, die von den reinen Isomeren bei der Konzentration $c$ induziert werden. Der Quotient $c_{\mathrm{cis}}^{\mathrm{o}} / c$ beschreibt den zur Zeit $t=0$ vorhandenen auf die Einwaagekonzentration $c$ bezogenen Anteil des cis-Isomeren.

Ausgehend von dem an der reinen trans-Verbindung 3 b gemessenen MTP-Wert (Tab. 1) wird die Ganghöhe $p_{\text {trans }}$ berechnet und der mit den Meßwerten gebildete Ausdruck $\ln \left|\left(1 / p-1 / p_{\text {trans }}\right)\right|$ gegen die Zeit $t$ aufgetragen. Die berechneten Werte liegen recht gut auf einer Geraden, wie gemäß Gl. (1) zu erwarten ist. Aus dem Anstieg ergibt sich eine Geschwindigkeitskonstante von $k=0,012 \mathrm{~min}^{-1}$.

Unter der Annahme, daß der an der chemisch isolierten Verbindung $4 \mathrm{~b}$ gemessene und in $\mathrm{Abb}$. 4 eingetragene MTP-Wert von $\beta_{\text {cis }}=-874 \mathrm{~m}^{2} / \mathrm{mol}$ dem der reinen cis-Verbindung entspricht, ergibt sich aus dem Achsenabschnitt ein cis-Anteil zur Zeit $t=0$ von $c_{\text {cis }}^{\circ} / c=86 \%$. Allerdings dürfte der tatsächliche cis-Anteil unter diesem Wert liegen, da anzunehmen ist, daß auch bei der Messung an der chemisch isolierten cis-Verbindung - wie bereits er-

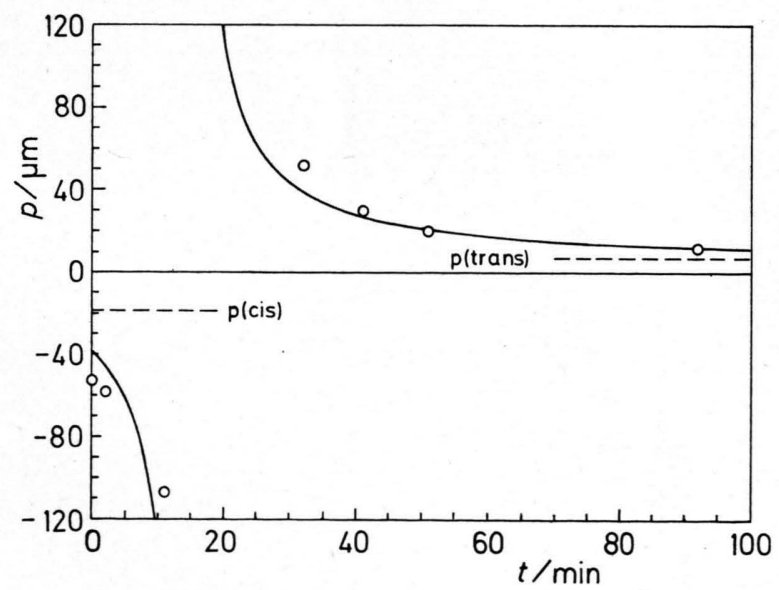

Abb. 6. Zeitlicher Verlauf der Ganghöhe bei der cis-transIsomerisierung der ortho-substituierten Azoverbindung 4 b nach $\mathbf{3} \mathbf{b}$. wähnt - ein geringer trans-Anteil in der Probe vorgelegen hat.

Der mit den so erhaltenen Werten für die Geschwindigkeitskonstante und dem cis-Anteil zur Zeit $t=0$ nach Gl. (1) berechnete zeitliche Verlauf der Ganghöhe bei der Isomerisierung der Azoverbindung $4 \mathbf{b}$ nach $\mathbf{3} \mathbf{b}$ ist im Vergleich zu den Meßwerten in Abb. 6 dargestellt. Die zu beobachtenden Abweichungen beruhen möglicherweise darauf, daß neben der thermischen Isomerisierungsreaktion auch eine photochemische Reaktion durch die intensive Mikroskopbeleuchtung abläuft, die aber wegen der variierenden Schichtdicke [10] ungleich über den Bereich der Probe erfolgt.

Die hier beschriebene Untersuchung der Kinetik veranschaulicht darüber hinaus die Schwierigkeiten bei der Bestimmung der MTP-Werte der reinen cisVerbindungen. Beispielsweise hätte sich die reine cis-Verbindung $4 \mathrm{~b}$ unter den vorliegenden Versuchsbedingungen bereits nach ca. $13 \mathrm{~min}$ auf den bei $t=0$ (Abb. 6) gefundenen trans-Anteil angereichert.

\section{Zusammenfassung}

Die hier durchgeführten Untersuchungen zeigen, daß der odd-even-Vorzeichenregel $[6,7]$ für die Induktion cholesterischer Phasen durch Moleküle mit chiraler Seitenkette ein größerer Gültigkeitsbereich zukommt, als zunächst anzunehmen war.

Die in cis- und trans-Konfiguration untersuchten ortho-substituierten Azobenzole sind die ersten Beispiele für Verbindungen, die trotz gleicher absoluter Konfiguration des chiralen Zentrums allein durch Änderung der Molekülkonfiguration von cis nach trans eine reversible Umkehr des Drehsinnes der im Flüssigkristall induzierten Helix bewirken.

Prinzipiell ergibt sich aus der hier beschriebenen photochemischen Beeinflussung der Ganghöhe die Möglichkeit, beispielsweise unter Ausnutzung des cholesterisch-nematischen Phasenumwandlungseffektes, Bildwandlerzellen aufzubauen. Ob dem auch praktische Bedeutung zukommt, muß weiteren Untersuchungen vorbehalten bleiben.

Diese Arbeit wurde aus Mitteln des Interdisziplinären Forschungsprojektes Flüssigkristalle der Technischen Universität Berlin gefördert. Wir danken der Gesellschaft von Freunden der TU für ihre finanzielle Unterstützung. 
[1] P. G. de Gennes, The Physics of Liquid Crystals, Clarendon Press, Oxford 1974.

[2] C. S. Bak u. M. M. Labes, J. Chem. Phys. 62, 3066 (1975).

[3] H. Stegemeyer, Ber. Bunsenges. Phys. Chem. 78, 860 (1974).

[4] W. Kuhn, Z. Elektrochem. 56, 506 (1952).

[5] H. J. Krabbe, H. Heggemeier, B. Schrader u. E.-H. Korte, Angew. Chem. 89, 831 (1977).

[6] G. W. Gray u. D. G. McDonnell, Mol. Cryst. Liq. Cryst. Letters 34, 211 (1977).

[7] G. Heppke u. F. Oestreicher, Z. Naturforsch. 32a, 899 (1977).
[8] B. W. van der Meer u. G. Vertogen, Z. Naturforsch. 34 a, 1359 (1979).

[9] G. Heppke, H. Marschall, P. Nürnberg, F. Oestreicher u. G. Scherowsky, Chem. Ber. 114, 2501 (1981).

[10] G. Heppke u. F. Oestreicher, Mol. Cryst. Liq. Cryst. Letters 41, 245 (1978).

[11] Zur photochemischen bzw. thermischen Isomerisierung des unsubstituierten Azobenzols in cholesterischen Flüssigkristallen vergleiche: E. Sackmann, J. Amer. Chem. Soc. 93, 7088 (1971). J. M. Nerbonne u. R. G. Weiss, J. Amer. Soc. 100, 5953 (1978). 